\title{
Goal and Risk Factors in Offshore Outsourced Software Development From Vendor's Viewpoint
}

\author{
Shareeful Islam ${ }^{1}$, Md. Mahbubul Alam Joarder ${ }^{2}$ and Siv Hilde Houmb ${ }^{3}$ \\ ${ }^{1}$ Software \& Systems Engineering, Technische Universität München, Germany \\ ${ }^{2}$ Institute of Information Technology, University of Dhaka, Bangladesh \\ ${ }^{3}$ Connected Objects Laboratory, Telenor R\&I Service Platforms, Norway \\ islam@in.tum.de,joarder@univdhaka.edu,siv-hilde.houmb@telenor.com
}

\begin{abstract}
Reducing production cost is vital for ensuring sustainable competitive strength. This is particularly true in software development, in which there has been a move from in-house development to global and now also to offshore-outsourced software development. In offshore outsourcing, development activities are mostoften moved to low-cost development environments that are locally managed. However, this type of outsourcing is not without problems. Most development projects are complex, and moving control and responsibility away from the client increase complexity. But, there is a trade-off between cost and complexity and control, as well as an increased chance of failure of the project. This paper contributes to identify the goals from the early development components and risk factors threatening the goals to fulfill. A goal-driven software development risk management modeling (GSRM) propose to supports this task. We conducted a study based on Delphi survey process to obtain the goals and the risk factors in a different cultural environment for the offshore vendors in Bangladesh
\end{abstract}

Keywords: Software Development Risk, Risk Management, Risk Modeling, Goal-Driven Modeling, Offshore Outsourced Software Development.

\section{Introduction}

Software development projects need to stay within budget, be innovative and deliver according to the endusers expectations to sustain the today's highly competitive market with short product lifetime. As many projects run over budget, reducing production cost is essential. One often much means to cut cost is outsourcing. Outsourcing is a frequently used cost reduction means, such as offshore-outsourced software development. However, Offshore Outsourced Software Development (O-OSD) also poses challenges such as language barrier, cultural difference, indirect communication, etc influence potential risks on the software development activities. Some of these risks are unique and subtle such as building trust across cultural boundaries, disputes due to different legal frameworks, loss of control of schedule and spending, and lack of understanding of the offshore environment. A recent ACM report suggested that outsourcing magnifies existing risk and creates new threats within the offshore project [1]. Therefore, risk relating to offshore software development must be assessed and controlled from early in the development process.

This paper presents the result of a survey investigating the threats to and the goals involved in successfully offshore-outsourced software development. The paper focuses on the top ten risks factors that obstruct the goals. The survey was conducted using a Delphi survey process and the participants in the survey were five software development companies in Bangladesh involved in offshore-outsourced development. The focus of the survey was to identify the goals and risk factors based on actual experiences in offshore projects. The survey context is from a developing country with limited IT infrastructure facilities, but where the offshore market is rapidly expanding through significant increase in investments in recent years. E.g., the European Union has ranked Bangladesh as one of the top 20 outsourcing destinations in the world [5]. Thus the identified risk factors from the survey are from a completely new cultural context than earlier studies, such as [6] [11] [14].

The paper also presents an approach to controlling risks and attaining goals in an offshore-outsourced development context called goal-driven software development risk management modeling (GSRM). The approach extends KAOS (Keep All Objective Satisfied) goal-modeling language [9] to accommodate software development risk management activities during requirements engineering phase. This facilitates to control risks from the early phases of development, as many projects fail due to incomplete or underspecified requirements. Furthermore, cost related to fixing errors during the testing phase is fifty times more than the cost of fixing these in the requirements phase [12].

The structured of the paper is as following. Section 2 presents some related work and outlines the main 
contributions. Section 3 outlines the survey method and Section 4 gives an overview of the results from the survey. Section 5 presents the goal-driven software development risk management modeling (GSRM) approach and finally, Section 6 concludes the paper and gives directions to future work.

\section{Related Works and Main Contributions}

In [4], Boehm, one of the pioneers in the area of software risk management, proposes a risk driven spiral model consisting of an iterative set of activities. In [7], Karolak proposes an approach called Software Engineering Risk Management (SERIM) that combines four interconnected risk tree based on 81 risk factors group into three main risk elements: technology, cost and schedule. It was already consensus from the existing literature that the main activities for software risk management are risk assessment and control. The GSRM approach enhances by including explicit activities for goal identification and elaboration. The survey reported in this paper followed the questionnaire undertaken by Karolak and which the SERIM approach is based on.

In [8], Kontio describes the Riskit methodology, which focuses on identifying stakeholder goals and risks that threaten these goals. Risks are analyzed and prioritized by deriving scenarios, which is a non-trivial task, as a scenario involves several probabilistic elements. The GSRM approach also starts with identifying the goals for successful software development, and in this perspective GSRM is similar to the Riskit approach. However, GSRM only deals with early technical and non-technical development components to assess and manage software development risk during requirements engineering phase.

In [2], Ansar et al. propose a Tropos goal risk framework that extends the Tropos goal model. The framework model and reason risk by the three layers: goal, event and treatment. However, no evidence show how the risks are prioritized based on the early development components.

In [6], Iacovou et al. summarize the top ten risk factors for offshore-outsourced development projects. Aspray et al. [1] provides an ACM task force report that considers risk from both technical and nontechnical issues. Furthermore, Tsuji et al. [11] propose a questionnaires assessment scheme considering software, vendor and project properties to quantify risk of offshore software outsourcing. The survey result shows the degree of importance among these properties and concludes that vendor property such as communication and project management ability mainly affect the result of development whereas software property such as requirement volatility did not affect the result. Sheng et al. [14] used similar analysis methods and conclude that project property is the main item affecting the success and that risk is culture dependent. The survey reported in this paper also identified culture as one of the important risk factors, but our study show that requirement volatility affects on the development activities.

\section{Survey Method}

The aim of the survey was to identify goals and risk factors involved in offshore-outsourced software development and to rank the risk factors. Due to the space restrictions, only a short overview of the survey and its results are presented here. The method followed in the survey was a three round Delphi process [13]. The main advantage of the Delphi method is that it facilitates multi-phase iterative surveys with controlled feedback loops. Phase 1 involved discovering the goals involved from the perspective of project success and high-level obstacles to these goals. Phase 2 focused on identifying risk factors by e.g. refining the high-level obstacles identified in phase 1 . These risk factors were then ranked in phase 3 .

The survey was conducted by interviewing five Bangladeshi software vendor companies involved in software development for its offshore client. Phase 1 also included collecting background information on the companies. The third phase was carried using a questionnaire that distributed electronically using email. A total of fifteen participants were involved in phase 3. The survey was carried out between November 2008 and January 2009.

\subsection{Phase 1 - Discover Goals and Obstacles}

The first phase used brainstorming with open ended question to elicit the main goals of offshore software development activities from the perspective of project success and any challenges involved, here referred to as high-level obstacles. In addition, a short profile of the vendor companies was outlined. The brainstorming started by identifying a set of general goals, based on existence literature [9] [10] [12], from the perspective of project success. These general high-level goals were then used as a starting point to discuss offshoreoutsourcing specific goal elaboration and refinement. The brainstorming phase facilitated the participants shared their assumptions and experiences from offshore projects. Note that the brainstorming was carried out separately at each of the five vendor companies involved in phases 1 and 2. And the participants within specific vendor company arrived at a common agreedupon set of goals at the end of phase 1. Table1 gives an overview of the background information gathered on the participating companies 
Table 1. Information about the companies

\begin{tabular}{|l|l|}
\hline Item & Information \\
profile & $\begin{array}{l}\text { Total number of employees is more than 50 } \\
\text { for four of the companies and less than } 20 \text { for } \\
\text { the fifth. }\end{array}$ \\
$\begin{array}{l}\text { Each company has more than three years of } \\
\text { experience with offshore software } \\
\text { development projects. }\end{array}$ \\
\hline Projects & $\begin{array}{l}\text { Share of total development activities and } \\
\text { coverage: 40\% of the projects targeted the } \\
\text { complete product and all development } \\
\text { activities, 35\% of the projects involved only } \\
\text { coding, 10\% of the projects involved only } \\
\text { testing and } 14 \% \text { of the projects involved only } \\
\text { maintenance. }\end{array}$ \\
\hline Participants & $\begin{array}{l}\text { 3 participants inhibiting both roles of director } \\
\text { and system developer, 5 participants had the } \\
\text { role of project leaders, 4 participants had the } \\
\text { role of software engineers, 2 participants had } \\
\text { the role of testers, and 1 participant had the } \\
\text { role of architect. } \\
\text { Average job experience of the participants in } \\
\text { offshore project is more than 2.5 years. }\end{array}$ \\
\hline
\end{tabular}

\subsection{Phase 2 - Identify Risk Factors}

A total of 82 close questions were prepared for the interview rounds in phase 2 with three possible answers. These questionnaires were based on the feedbacks from phase 1 and aimed at facilitating the refinement of the goals and the high-level obstacle. In addition, there was an open question aimed at capturing any missing risk and any information not addressed in the questionnaires. The open question also had the role to reduce the bias of the closed questions, as it gave the participants an opportunity to give feedback on issues they had not been able to express thus far. The participants were also asked to rank the risk factors internally. The interviews using the questionnaires lasted for about two hours per participants. At the end of each interview, the participant was asked to narrow down the risk factors. We produced a consolidated rank of risk factors based on mean rank of individual risk factors and associate standard deviation. Note that at this stage the interview mainly focused on identifying the risk factors.

\subsection{Phase 3 - Rank Risk Factors}

In the final phase the participants was presented by the risk factors with their own rating and the consolidated ranking. The participants were then asked to reconsider their rankings and to provide a final ranking for the identified top ten risk factors. We received five responses from five vendors because all participants of the same company jointly selected the top ten risks. This enabled them to reevaluate their previous opinions, so that the result moved toward prefect consensus [13], and the risk factors were linked to the sub-goals that they may affect.

\section{Results from the Survey}

The result of the survey (interviews and questionnaire) showed a high consensus on five of the identified high-level goals: (1) Attain project factor, (2) Manage human factor, (3) Manage organizational factors, (4) Attain information security, and (5) Compliance with legal issues. These high level initial goals were refined into sub-goals by the participants as part of the information collection activities in phase 1, and internally ranked according to the scale (1-5), where 1 means highest priority, by means of their priority in contributing to a successful project. In phase 2 , risk factors were identified and aggregated into a consolidate set based on the participants experience from both successful and failed offshore-outsourced development project. These risk factors were then ranked according to the quantitative scale $[1,10]$ in phase 3 , where the values from 1 to 6 refers to various degree of 'very important', and the values from 6 to 10 refers to various degrees of 'important', in terms of posing a risks to arriving at a successful project. The risk identification focused on the factors relating to the offshore-outsourced software development environment. The reason for this is that the risk factors that are influenced by external parameters, such as local infrastructure facilities and culture, interaction with the client, usually require a longer time to fix and are thus usually ranked higher than the risk factors that can be addressed locally at the offshore location. At the end of phase 3, the risk factors were linked to the subgoals using a cause-consequence analysis. Table 2 shows the identified top ten risk factors and the subgoals that these obstruct.

To understand Table 2, it is necessary to look into the identified sub-goals from phase 1. Taking the goal: 'Attain project factor', assigned as the highest priority in phase 1, focusing on development process, final software system, time and budget from the perspective of project success, was refined into six sub-goals: (1) clear business specification and system vision, (2) stay in budget, (3) maintain realistic schedule, (4) attain technical feasibility, (5) effective development process, and (6) attain product quality. In general, early in the requirements phase, initial elicited development artifacts such as business specification (e.g., business goal, domains, business process, future end-user, and business rules), system vision, and user and system requirements require to review for fulfillment of these sub-goals. Furthermore, project-related information such as schedule, cost, project management capability etc also influences the ability to attain the sub-goals. The risk ranking and linking to sub-goals in phase 3 identified two very important risk factors: (2) unstable 
requirements and (4) ambiguous requirements. This makes sense, as clear understanding and freezing of requirements are difficult to achieve in general, and particularly in an offshore development context. The reasons are lack of direct face-to-face communication, lack of knowledge and understanding of the end-users and the business context of the client, and ineffective requirements elicitation with little interaction with the client. Local environmental factors such as interrupted network service, electricity problem, and strike (10) obstruct stay under budget sub-goal. Although according to the participants experienced this risk rank is not very important as compare to the risk factors within 1 to 5 . In addition, some of the offshore companies have implemented specific strategies to pull the project through despite low profits. This tendency, along with some unwanted costs (9), poses obstruct the goal 'Stay in budget' as shown in Table 2.

Table 2. The top ten risk factor

\begin{tabular}{|c|c|c|}
\hline Rank & Risk Factor & Sub-Goal \\
\hline 1 & $\begin{array}{l}\text { Lack of involvement from } \\
\text { client in development } \\
\text { activities }\end{array}$ & $\begin{array}{l}\text { Effective } \\
\text { involvement }\end{array}$ \\
\hline 2 & Unstable requirements & $\begin{array}{l}\text { Reduce error from } \\
\text { requirements }\end{array}$ \\
\hline 3 & $\begin{array}{l}\text { Lack of communication } \\
\text { ability and coordination } \\
\text { possibilities }\end{array}$ & $\begin{array}{l}\text { Effective } \\
\text { communication and } \\
\text { coordination }\end{array}$ \\
\hline 4 & Ambiguous requirements & $\begin{array}{l}\text { Reduce error from } \\
\text { requirements }\end{array}$ \\
\hline 5 & $\begin{array}{l}\text { Lack of important domain } \\
\text { knowledge of staff }\end{array}$ & $\begin{array}{l}\text { Quality and relevance } \\
\text { of staff }\end{array}$ \\
\hline 6 & $\begin{array}{l}\text { Lack of commitment and } \\
\text { capability among } \\
\text { management }\end{array}$ & $\begin{array}{l}\text { Proper management } \\
\text { direction and support }\end{array}$ \\
\hline 7 & $\begin{array}{l}\text { Lack of ability to } \\
\text { effectively incorporate and } \\
\text { in general handle change } \\
\text { in management }\end{array}$ & $\begin{array}{l}\text { Effective } \\
\text { development } \\
\text { activities }\end{array}$ \\
\hline 8 & $\begin{array}{l}\text { Employees not showing } \\
\text { up for work }\end{array}$ & $\begin{array}{l}\text { Quality and relevance } \\
\text { of staff }\end{array}$ \\
\hline 9 & $\begin{array}{l}\text { Failure to early identify } \\
\text { hidden costs and extra } \\
\text { expenses }\end{array}$ & Stay in budget \\
\hline 10 & $\begin{array}{l}\text { Failure to consider factors } \\
\text { that delays works, such as } \\
\text { worker strike, interrupted } \\
\text { network service and } \\
\text { power supply }\end{array}$ & $\begin{array}{l}\text { Maintain } \\
\text { schedule }\end{array}$ \\
\hline
\end{tabular}

Looking at the goal assigned the second highest priority, 'Manage human factor', this was refined into four sub-goals: (1) quality and relevance of staff, (2) effective communication and coordination, (3) proper management direction and support, and (4) effective client involvement by mainly focusing on the nontechnical issues within early development environment. As can be seen in Table 2, all these sub-goals are affected by the top ranked risk factor and thus of great importance for succeeding with an offshore development project. Several of the participants in the survey mentioned explicitly that there were often lack of involvement and effective communication with the client. The consequence of this was noted to be rework, conflict between client and vendor, and sometimes it also resulted in wrong assumption, and by that inadequate requirements. In fact, all participants stated that they had faced this risk in most of their offshoreoutsourced development projects and agreed to rank lack of client involvement as the top risk factor (1). Furthermore, lack of communication ability and coordination possibilities (3) by the vendor is also a risk factor that directly influences effective communication and coordination. It was revealed that this was mainly due to language and cultural barrier, as well as lack of direct (face-to-face) communication. Project member's lack of domain knowledge (5) is also very important risk factor mainly due to incomplete understanding of client business needs. Almost every participant agreed that there is always adequate technical expertise within the vendor site. Lack of management commitment and capability (6) due to inadequate experience and professionalism often jeopardize the overall project success and hinder the goal proper management direction and support

The third highest prioritized goal was 'Manage organizational factors'. This goal focuses on managerial issues rather than technical issues and refined into four sub-goals: (1) effective risk culture, (2) stability of the organization, (3) adequacy of the development facilities and resources, and (4) effective policy and procedure. Risk factors that influences meeting this goal are lack of management capability (6), and employees not showing up for work (8) (see Table 2).

In addition to the three above-described high-level goals, two more goals were identified: 'Attain information security' and 'Compliance with legal issues' in phase 1. However, no direct risk factors were identified for these goals because existence local legal infrastructure does not obstruct the O-OSD environment. However, some participants mentioned that variation of bank fee sometimes create difficulties to transfer money from a foreign country. No participants faced problem with security and hence they are not discussed further in this paper.

\section{Goal-Driven Software Development Risk Management Modeling (GSRM)}

To effectively address risks for the successful offshore-outsourced development projects up-front it is useful to explicitly model the relations between the high-level goals and the risk factors. Risks are addressed by employing some cost effective control action, and as one treatment may address several risk factors and hence high-level goals, it is also important 
to model the relationship between treatments and goals. Goal-driven modeling can be used in this context and this paper proposes a Goal-driven Software development Risk management Modeling (GSRM) approach for managing risk in offshore-outsourced development.

Goal and risk are complementary entities in any software project. There will be no risk if there are no goals or expectations [8]. Therefore, risk management requires identifying, reviewing, and refining the goals. Goal oriented requirements engineering methodology (e.g. KAOS, $\mathrm{i}^{*}$, Tropos) has long been recognized as essential for eliciting, analyzing, and validating requirements. Nonetheless, the methodologies do not consider software development risks during requirements phase except [2]. We extend the KAOS goal modeling language [9] to support early stage risk management activities. KAOS defines obstacle as a construct that can be used to identify undesirable behavior against the strategic interest of a stakeholder [9]. GSRM have adopted this construct and defines software risks as obstacles that contribute negatively to fulfill specific development goal. These risks must be addressed and GSRM does this by assigning suitable treatment actions (remember we are concentrating on the requirements phase, so treatment actions must be related to requirements activities). GSRM adopts goal and obstacle concept from the KAOS and further extends with risk assessment and treatment for managing software development risk. Furthermore, GSRM facilitates the reasoning and tracing of treatment actions and their ability to mitigate risks, and hence, to fulfill goals. This is done using the four layer modeling structure of GSRM: (1) goal, (2) risk obstacle, (3) assessment, and (4) treatment layer.

\section{Goal layer}

The goal layer identifies the main factors that contribute to the success of the development project. Successful offshore-outsourced software development depends on a number of goals as has been discussed earlier in this paper, such as attain business objectives, error free requirements, active client involvement, accurate time and budget estimation, quality of staff, etc. A holistic view of all these success factors is required to identify and fulfill development goals [3] [10] [12]. The goals involved in the development activities must be achieved, maintained, ensured, managed and reduced [10] to carry out a successful development project. Also, the goals must be formulated such that they directly addresses the important aspects in the sense that its related features, properties and constraints need to be eventually satisfied for the goal to be fulfilled. Goals can be stated at different levels of abstraction from coarse-grained to finer-grained goals, as the risk factors is linked via the sub-goals to the higher level goals.

\section{Risk obstacle layer}

Risk obstacle layer identifies the potential software risk factors as obstacles that obstruct these goals. Obstacles are the opposites of the goals (i.e., undesirable goals). Therefore, the obstacle categories should be related to and derive from the goal categories. Software risk is an example of an obstacle that may prevent the goal from being achieved or maintained. Risk obstacle layer supports the risk identification activities by linking the obstacles to the goals. Same risk obstacle can be relevant to more then one goal. Examples of obstacles that generally pose risks in an development environment are missing business specification, lack of understanding between the involved parties, ambiguous requirement, and ineffective development process.

\section{Assessment layer}

The assessment layer is used to precisely annotate the individual risk obstacle. The main purpose of this layer is to analyze the risk event caused by the risk factor. Each risk event characterized with two properties: likelihood and severity. Same risk factor can pose more than one risk event as well as same risk event can obstruct more than one goal. This representation allows to model situation where an event influences by more than one risk factor and at the same time negatively impacts on single or several goals. An obstruction link is established from risk event to the specific goal it obstructs. The assessment layer also prioritizes the risk factors based on the likelihood and severity of impacts. In GSRM, risk analysis explicitly considers the risk events having only negative impact to the goals from the early development components.

\section{Treatment layer}

Finally, the treatment layer models possible control actions and are used to assist in deciding on suitable treatment strategies. The aim of this layer is to control risks as early as possible by assigning appropriate countermeasures. Different strategies can be followed to control the risk. However, the first treatment step is always to reconsider the risks such that no unnecessary treatments are assigned. Furthermore, no control action should be assigned to low impact risk. This is a strategy that is followed in most risk assessment methods and means that the identified risk factors are prioritized and treated based on their potential impacts.

Potential treatment strategies are preventing risk, reducing risk, avoiding risk, transfer risk, and accept risk. In cases where it is not possible to avoid the risk all together, it should be investigated whether it is possible to reducing the likelihood or severity of the risk. When neither preventing nor reducing risks is a possible option, it might be necessary to transfer or accept the risk. Additionally it is also beneficial to 
investigate the costs and opportunities before assigning a control action. In GSRM, we establish a link from control action to goal as contribution link to support the tracing. Figure 1 shows the different layers of GSRM. Note that GSRM uses the same notations for goal obstacles and treatment as the KAOS model.

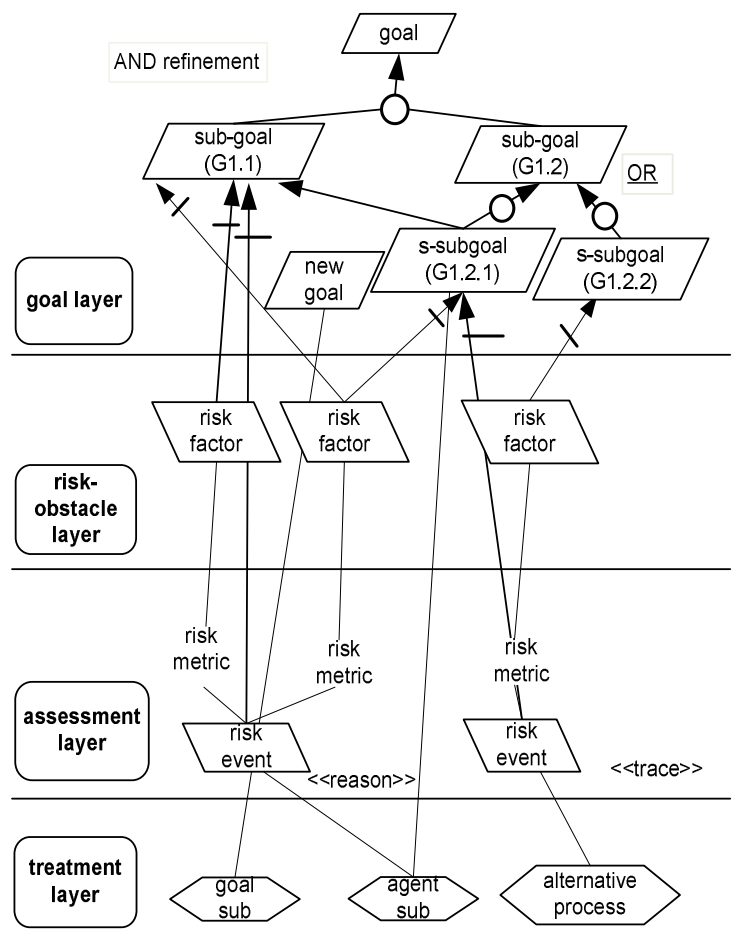

Figure 1. Overview of GSRM for offshoreoutsourced software development

\section{Conclusions and Further Direction}

The paper presents the method used and results from a survey aiming at identifying the main success criteria (goals) and risks that obstruct these goals in offshore-outsourced software development. This survey is a part of ongoing research on the Goal- driven Software development Risk management Modeling (GSRM). The aim of the work is to arrive at as-muchas possible pre-filled GSRM to easy the assessment of risks in the early phases of offshore-outsourced development. Thus far it seems reasonable to focus on goals and risk factors by means of risk assessment and treatment assignment activities that directly feeds information into the goal model. It has also been some promising results of using GSRM model to mange risks starting in the requirements phase. However, the work is still at the early stages and further work is needed to conclude on the feasibility, effectively, and scalability of the GSRM approach.

Future work includes a replication of the survey and to apply the GSRM approach to support offshore- outsourced development in practice at one of the companies involved in the survey presented in this paper. Future work also involves establishing a set of guidelines for the ICT Ministry in Bangladesh based on the survey results with the aim for the Government of Bangladesh to better support the emerging offshoreoutsourced development environment in Bangladesh.

\section{Acknowledgement}

This research is partly supported by the Telenor COLAB - a part of the Telenor Connected Object project.

\section{References}

[1] Astray, W., Maydays, F., and Verdi, M.Y. Globalization and off-shoring of Software: A Report of the ACM Job Migration Task Force, ACM, NY, 2006.

[2] Ansar, Y., and Georgina, P. Modeling Risk and Identifying Countermeasure in Organizations, In Proceedings of the first International Workshop on Critical Information Infrastructures Security, pp. 55-66, Springer, 2006.

[3] Islam, S. and Dong, W. 2008. Human factors in software security risk management, In Proceedings of the first international Workshop on Leadership and Management in Software Architecture. LMSA '08, In ICSE 08, ACM, New York, NY, 13-16.

[4] Boehm, B. Software Risk Management: Principles and Practices, IEEE Software, Vol. 8, pp. 32-41, January 1991.

[5] Bangladesh Associate of Software \& Information Services (BASIS), http://www.basis.org.bd(last accessed March 2009).

[6] Iacovou, C. L. and Nakatsu, R. A Risk Profile of Offshore-outsourced development project, Communication of the ACM, Vol 51, No. 6, June 2008.

[7] Karolak, D. Software Engineering Risk Management, IEEE Computer Society Press, CA, USA, 1996.

[8] Kontio, J. The Riskit Method for Software Risk Management, Version 1.00, Technical Report CS-TR3782/UMIACS-TR-97-38, University of Maryland, Computer Science at College Park, Maryland, USA, 1997.

[9] Lamsweerde van A. Requirements Engineering: From System Goals to UML Models to Software Specifications, Wiley, January 2009.

[10] Ropponen, J. and Lyytinen, K. Component of Software Development Risk: How to address them? IEEE Transactions on Software Engineering, Vol.26, Issue: 2, Feb 2000.

[11] Tsuji, H., Sakurai, A., Yoshida, K., Tiwana, A., and Bush, A. Questionnaire-Based Risk Assessment Scheme for Japanese Offshore Software Outsourcing SEAFOOD 2007, LNCS 4716, pp. 114-127, Springer, 2007.

[12] Procaccino, J. D, and Verner, J. M. Case Study: Factors for Early prediction of software development success; Information and Software Technology; Vol. 44, pp. 53-62, 2002.

[13] Schmidt R., Decision Sciences, 28(3), pp. 763-774, 1997.

[14] Sheng Z.,Nakano M.,Kubo S.,Tsuji H., Risk Bias Externalization for Offshore Software Outsourcing by Conjoint Analysis, New Frontiers in Artificial Intelligence, Volume 4914/2008. 